\title{
Generic Homo sapiens and Unique Mus musculus: Establishing the Typicality of the Modeled and the Model Species
}

\author{
Barbara L. Finlay \\ Behavioral and Evolutionary Science Group, Department of Psychology, Cornell University, Ithaca, NY, USA
}

\section{Keywords}

Allometry - Brain development · Brain evolution .

Comparative neuroanatomy $\cdot$ Cortex $\cdot$ Evolution of nervous systems · Humans

\begin{abstract}
The question of how complex human abilities evolved, such as language or face recognition, has been pursued by means of multiple strategies. Highly specialized non-human species have been examined analytically for formal similarities, close phylogenetic relatives have been examined for continuity, and simpler species have been analyzed for the broadest view of functional organization. All these strategies require empirical evidence of what is variable and predictable in both the modeled and the model species. Turning to humans, allometric analyses of the evolution of brain mass and brain components often return the interesting, but disappointing answer that volumetric organization of the human brain is highly predictable seen in its phylogenetic context. Reconciling this insight with unique human behavior, or any species-typical behavior, represents a serious challenge. Allometric analyses of the order and duration of mammalian neural development show that, while basic neural development in humans is allometrically predictable, conforming to adult neural architecture, some life history features deviate,
\end{abstract}

notably that weaning is unusually early. Finally, unusual deviations in the retina and central auditory system in the laboratory mouse, which is widely assumed to be "generic," as well as severe deviations from expected brain allometry in some mouse strains, underline the need for a deeper understanding of phylogenetic variability even in those systems believed to be best understood.

(c) 2019 S. Karger AG, Basel

\section{Introduction}

Model organisms are used in two distinct ways in evolutionary neuroscience. The first use focuses on singularities - a single species is chosen for its unique adaptations or unusual capacities. On this research path, we can include the study of ourselves, where we try to understand our most important attributes in terms of their evolution. The second use of model organisms is to exploit their generic features to investigate basic organizational principles. From Caenorhabditis elegans to Drosophila melanogaster to Mus musculus and Rattus rattus, we now expect to find common organizational themes across the brain mechanisms and behavioral abilities not only between closely related species, but across phyla. Near 50 years ago, Nauta and Karten [1970] produced a prototypical

\section{KARGER}

(c) 2019 S. Karger AG, Basel

E-Mail karger@karger.com

www.karger.com/bbe
Barbara L. Finlay

Department of Psychology, Cornell University

248 Uris Hall

Ithaca, NY 14853 (USA)

E-Mail blf2@ cornell.edu 
vertebrate brain for this generic beast, which they called the "shmoo brain," and have been reasonably well rewarded in their hope. These separate uses of models emphasizing uniqueness or typicality have both been productive, and the language associated with the promotion of each is second nature to neuroscientists questing for research grants. From time to time, though, it is a good exercise to examine basic research customs and assumptions. This short review makes no pretense of being a comprehensive historical account or a close analysis of the research strategies which eventually generated thousands of researchers and their studies. Rather, the intention is to bring the features of initial research choices back to awareness, and to promote discussion with an occasional provocative claim.

\section{Singularities}

When in search of singularities, what makes an attractive feature? The study of neuroethology was founded on a collection of strategic guesses about how best to gain entry to and understanding of sensorimotor organization in the brain [Hoyle, 1984]. Much of this work has involved the deliberate choice of exotic animals with specialized foraging or communication systems such as echolocating bats [Suga, 1989], electroreceptive fish [Hopkins, 1980], or croaking frogs in an amorous state [Capranica and Rose, 1983]. These sensory systems would confound the use of introspective hunches about our own sensory mechanisms as a research starting point. Rather, close measurement, induction, and analysis are demanded from the outset. Using highly specialized animals was a further bet that the system under study was under strong adaptive pressure, thus preventing the accidental waste of time on a mechanism not optimized for its specific function. A pervasive presumption of this approach was that all components of a specialized system, both peripheral and central components, were invariant and genetically specified.

A complementary research strategy chose species that possess some feature that is analogous to human special abilities, independent of phylogenetic relatedness, like color vision [Bowmaker, 1998], facial recognition [Sheehan and Tibbetts, 2011], or vocal communication. For example, because of the relative absence of learned vocal communication in terrestrial mammals, bird song has for years been a model for multiple facets of human language [Nottebohm et al., 1976]. These studies have revealed similarities in early learning, critical periods, grammatical structure, laterality in brain representation, and even genetic mechanisms [Doupe and Kuhl, 1999]. Without intention, this strategy in studying bird song produced a peculiar outcome. At first (and still), there was little agreement about how the song-related nuclei of the bird forebrain should be named to reflect cross-vertebrate homologies. When the accuracy of the term hyperstriatum ventrale, pars caudalis (HVC) became contested, the researchers interested in the design features of vocal communication systems, rather than its homologies, gave the HVC the retro-acronym of "high vocal center" [Rasika et al., 1994]. Since the necessary tools to accurately capture bird-mammal homologies would not be available for many years [as in Jarvis et al., 2005], useful research time was likely gained through this sidestep. An unfortunate conjunction, however, arose with assumptions made by linguists, principally those of the Chomskyan school [Hauser et al., 2002]. Human language, for entirely separate reasons, had been viewed as a separate module genetically inserted in the human cortex, having no meaningful phylogenetic continuities with the learning or communication systems of non-human animals. As the nuclei of the song system acquired an idiosyncratic set of names that also suggested they were a separable module residing fairly independently in the bird brain, both research areas effectively disconnected themselves for many years from the rapidly advancing work on cross-vertebrate commonalities in motor and communication systems. Both birdsong and human language neuroscience have only rather recently begun the process of repair and reintegration into a more general understanding of vertebrate brain organization [e.g., Poeppel, 2014; Hisey al., 2018].

\section{Generic Behavior and Brains}

While a good amount of the research in animals with complex brains across all of phylogeny falls into the class of descriptive research in a comparative context, or the neuroethological tradition I just described, much of it aims to describe basic features of nervous system organization and structure for medical or analytic purposes in organisms whose relative simplicity may give an advantage. A substantial evolutionary leap of faith was originally required to suppose that basic features of nervous system organization even existed. The three phyla where more complex brains have emerged independently are the Mollusca (gastropods like the marine mollusk Aplysia and the cephalopods), Arthropoda (crustaceans like Limulus and the multiple insects including Drosophila), and Chordata (non-mammalian vertebrates like the zebra fish Danio rerio and the zebra finch Taeniopygia guttata, as well as mammals including $M$. musculus, $R$. rattus, and 
Macaca mulatta). The three phyla diverged over 450 million years ago, in or before the Cambrian, and their common ancestors have minimal nervous systems. Fundamental features of neuronal physiology were shown to be held in common early on, but commonalities of more elaborated features required demonstration, such as the argument made by Greenough and Bailey [1988] for parallel processes of learning and memory in both Aplysia and the lab rat. These commonalities retroactively justified the choice of these species, which were initially selected on the basis of accessibility and ease of laboratory maintenance.

The jaw-dropping demonstration that transplantation of the embryonic primordium expressing the HOX gene Pax 6 was capable of orchestrating the production of a species-typical eye in both Drosophila and the lab mouse [Quiring et al., 1994], when their likely common ancestor was not known to have any eye, was a turning point in the understanding of nervous system phylogeny and ontogeny. This observation was quickly subsumed into a more general understanding of a genetically conserved basis of the invertebrate and vertebrate body plan, as well as conservation of major features of cell and neuron physiology, and was a major stimulus to comparative research, and the generation of the field of evo-devo [Gerhart and Kirschner, 1997]. Sometimes, this enthusiasm appears to have gone too far - not long ago, an extensive, finicky list of similar characters was required to establish homology of complex phenotypes between phylogenetically distant animals, but now, arguments are more casual. For example, human-defined emotional states like anxiety are extended to fruit flies by description of a few functional similarities of the effects of repeated alarming stimuli on both [Gibson et al., 2015]. We do not yet have a comprehensive understanding of whether similarities in initial body plan and a roughly comparable adult function will dictate interesting homologies in developmental paths to adult structures, but that is a focus of much current research.

\section{Using the Generic to Study the Unique: Primate \\ Models}

Primates, from lemurs to chimps, are research subjects primarily because of their phylogenetic closeness to humans, not for their convenience as laboratory denizens: they are employed as examples of possible sources of human abilities. In addition to the first consideration, general braininess, primate curiosity, expressive eyes, and communicative style make the monkey offer convenience in trainability and accessibility. The pitfalls of such an approach are obvious when non-human primates are as- sumed to have less evolved versions of the features we find most interesting in humans, and the true diversity of the primate order is poorly appreciated. For example, Harlow's work on the outcome of poor mothering in "the monkey" was assumed to be a good model of human developmental pathologies and defined much of the early views of good parenting and "secure attachment," as well as the pathological outcomes of bad parenting. At the time, however, the field of primatology was hardly launched, and primates were not known to have multiple styles of parental care. As it turns out, parenting in rhesus macaque is one of many primate types of parental behavior, and quite different from human parenting, which matters a great deal for the outcome of pathological mothering [reviewed in Hawkes et al., 2017].

\section{What Is Unique and What Is Generic?}

The uniqueness or typicality of any particular trait in a model species is an empirical claim that is often assumed or asserted, and rarely tested. As the species that is usually modeled is Homo sapiens, the uniqueness of traits and their underlying mechanisms enters research strategies twice, in the human trait to be described and its state in the model species. In the remainder of this paper, two specific cases are contrasted. First, several claims about human uniqueness are examined, looking at the kinds of issues and errors that typically emerge, and what sort of information can inform interpretation. Second, the prototypical generic animal, the laboratory mouse, is examined for the ways in which it may be a poor choice for the human brain organization and capacities it is expected to model, and the ways it may be successful. Because of the singularity of $H$. sapiens, researchers have allowed themselves unusual license in speculation about our evolution, forgetting that any other particular species is equally singular. Better methods have, in fact, been developed to keep speculation productive and creative rather than fanciful by placing the human species and its models in a more informed phylogenetic context.

\section{Human Exceptionalisms, Present but Not Pervasive}

\section{The Allometry of Neural Mass and Neuron Number}

Whole Brain Allometry

In the perennial quest for the simplest diagnostic measure of human uniqueness, one has survived years of methodological assaults and database expansions (at least with respect to mature vertebrates, and with a blind eye to the smallest of those). That measure is relative brain 
mass with respect to body mass, encephalization [Jerison, 1973]. This brain/body contrast has been variously realized as a simple ratio, fancier ratios, or $z$-scores to reflect the populations being contrasted. Encephalization has been found to correlate with niche complexity and taxonomic features like endothermy [Northcutt, 1981], and real-world measures of behavioral complexity [Lefebvre et al., 2004; Sol et al., 2005; Lefebvre, 2013]. Considering absolute brain mass independent of body size, however, elephants and many cetaceans may have 2-5 times more brain volume than humans, so this measure cannot by itself dictate human-style cognitive sophistication. Even so, absolute brain mass studied for both cross-species and individual variation effects can often predict specific cognitive measures better than relative size [MacLean et al., 2014; Stevens, 2014; Nave et al., 2019]. In my own laboratory group's many attempts to predict features of brain organization, we found only one instance where encephalization outperformed absolute brain size in terms of explaining a measure's variance. Relative brain size predicted the number of cortical areas observed in the entire cortical sheet slightly better than that predicted by absolute brain size alone [Finlay et al., 2005]. By contrast, changes in absolute brain size produce multiple reconfigurations in the internal organization of brains [e.g., Cahalane et al., 2014].

The question of which of these gross measures can predict any aspect of behavioral complexity or cognitive abilities, when the question of which best places humans on top is put aside, may be a very ill-formed one [Healy and Rowe, 2007]. Especially if we assume that, working through development, evolution is able to fine-tune any feature of the brain in any location, from receptors to dendritic arbors, to numbers of neurons in a nucleus, to network features, and so forth, it may appear more profitable to examine only functional systems, and those in great detail. Which developmental features can actually be "accessed" by evolutionary selection on individual variation, however, remains an empirical question, and in some cases the number of features seems limited. For example, a single variable, absolute duration of development dominates variance in brain mass, at least in mammals [Workman et al., 2013; Halley and Deacon, 2017]. In turn, overall brain mass closely predicts volume and number of neurons from gross brain subdivisions down to much finer detail, via the allometric scaling described in the next section [Finlay and Darlington, 1995; Finlay et al., 2001, 2014; Finlay, 2008]. Therefore, we may have no choice but to continue to investigate macro- and micro-organization in parallel.

Evaluating Model Species
Allometric Predictability of the Neocortex

Looking at the brain by major subdivisions, it is now fairly well known that the human neocortex (or isocortex) is precisely predictable from whole brain mass by the allometric equations derived from all primates studied to date. It is disproportionate in its relative size because of its positive allometry with respect to the whole brain, and whole brain volume is largest in humans. The absolute size of the isocortex in humans is predicted from the allometric equation of cortex volume versus rest-of-brain derived from all primates [Jerison, 1973; Hofman, 1989]. We may expand this claim to span all mammals if we allow an adjustment for initial allocation of tissue to olfactory/limbic regions versus rest-of-brain, which varies across taxonomic orders [Finlay and Darlington, 1995; Reep et al., 2007]. The nature of allometric analysis will not be discussed here, but readers are directed to either of two recent reviews for a basic description and review of central issues and concerns, the first directed toward psychologists [Hawkes and Finlay, 2018] or the second for cell biologists or physiologists [Charvet and Finlay, 2018].

The significance of cortical predictability is underappreciated. It means that any gross brain division, cortex to medulla, is governed by the allometric equation for that division. There is no need to search for genetic mechanisms specific to the cortex to account for its volume, relative to other structures, even though this research question has been undertaken multiple times and identified many candidate genes [see reviews in Vallender, 2008; Wilsch-Bräuninger et al., 2016]. The obvious candidate for the mechanism specifying the size of the major brain divisions is the duration of neurodevelopment, which spans neurogenesis, establishment of intraregion axonal and dendritic connectivity, and myelination. This coordinating mechanism neatly ranks the allometric slopes of gross brain division volumes from medulla to neocortex and cerebellum [Workman et al., 2013]. Fifty years ago, genetic analyses of the control of brain and body mass were ongoing, in the framework of allometric analysis. For example, Lande [1979], in the paper "Quantitative Genetic Analysis of Multivariate Evolution Applied to Brain: Body Size Allometry" said “... it is not unreasonable to approach the analysis of evolutionary forces producing interspecific allometry in brain and body weights through quantitative genetic studies on the gross measures themselves, rather than using a finer subdivision of parts." Returning to this research path with the power of current genetic techniques would be illuminating.

Even if it is true that the human isocortex is not "special" or unique in the sense of allometric unpredictability,

Brain Behav Evol 2019;93:122-136 
Fig. 1. Log-scale plots comparing prefrontal cortex (PFC) gray and white matter volume with reference values across species (macaques, diamonds; chimpanzees, squares; humans, circles). Volumes of conservative PFC (red), non-PFC (black), and area V1 (green) gray matter plotted against total cortical gray matter volume. Volumes of area 4 are plotted with solid magenta markers without a corresponding linear fit. Solid lines represent the best fit using mean macaque, chimpanzee, and human data points; dotted lines represent 95\% CIs. Reprinted with permission from Donahue et al. [2018].
$\mathrm{PFC} /$ non-PFC/areal vs. total gray matter

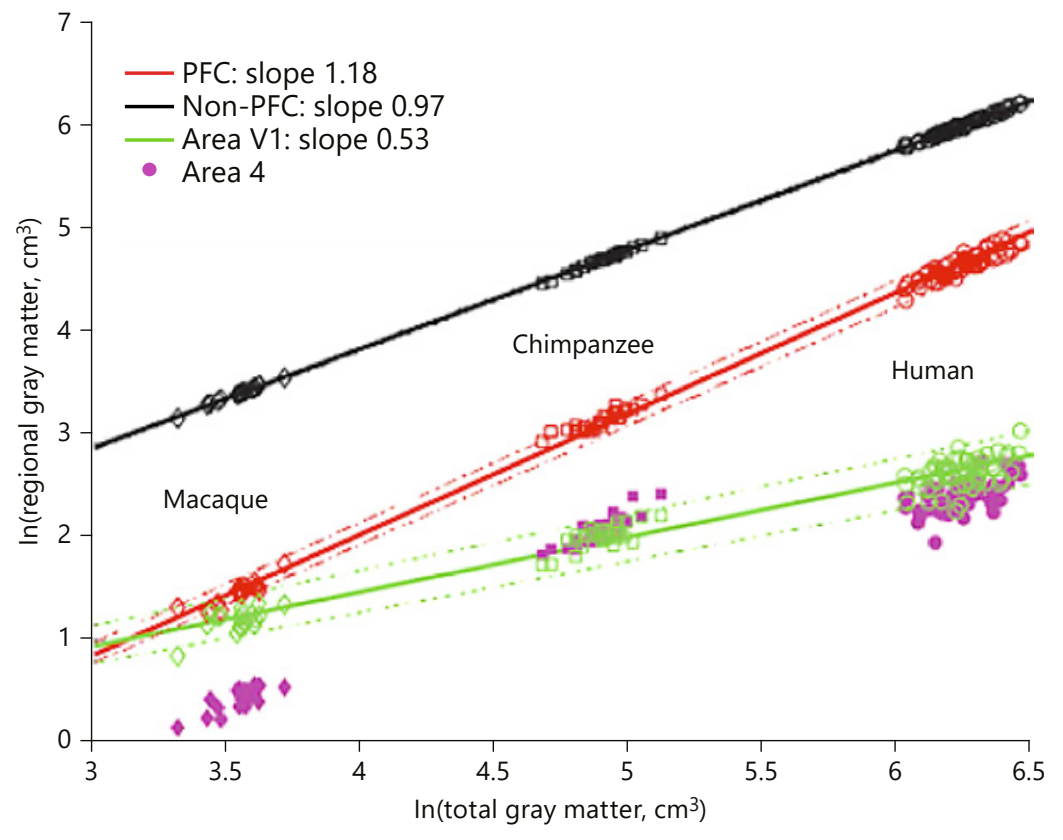

this insight cannot be the end of the story. The neocortex is the largest component of the human brain and most abilities essential for human success require it. Therefore, an account of the mechanisms that organize the cortex for human specific capacities is still needed. A recent debate about whether the prefrontal cortex is especially enlarged in humans exactly parallels the debate just described and provides hints about how such paradoxes might be resolved.

\section{Allometric Predictability of Prefrontal Cortex}

In a recent article reporting on prefrontal cortex scaling, using extensive MRI cortical measurements of multiple individuals in three primate species, Donahue et al. [2018] reported that "using tissue volume analyses, we show a disproportionately large amount of gray and white matter corresponding to PFC [prefrontal cortex] in humans compared with non-human primates." They give this rationale for their study: "Some studies report that the human PFC is disproportionately larger, whereas others report a more uniform scaling" (Fig. 1). In response, Barton and Montgomery [2019] correctly object that: "Contrary to their presentation of the debate, this fact [disproportionately large prefrontal cortex in humans] is uncontroversial. However, proportional size is often a misleading functional metric because of nonlinear, or allometric, scaling ..." No researcher in fact has argued or presented evidence that the frontal cortex has more uniform scaling with respect to the rest of the cortex, by which Donahue et al. [2018] seem to mean a linear or isometric scaling, the frontal cortex enlarging at the same rate as the remaining cortex, as you would see when enlarging a photograph. The debate concerned whether the prefrontal cortex in humans is even larger than its already substantial positive allometry would predict [Barton and Venditti, 2013; Passingham and Smaers, 2014]. Though linear scales are the ones we are most comfortable with by virtue of how mathematics is taught, there is nothing basic or intrinsically natural about them. Linear scaling is a rather rare phenomenon in biological systems, as most biological processes like cell division, network growth, energy and heat requirements with changing mass and so forth are power functions [West et al., 1997]. Occasionally, a particular physical constraint can require linear scaling - for example, eyes of different diameters scale linearly and be rendered superimposable by magnifying one or the other to match because the relevant optics scale linearly [Remtulla and Hallett, 1985]. Such examples are not common. 
The common mistake of taking predictable allometric scaling for a special adaptation in a species of interest, particularly at the extremes of distributions where disproportionality is the most noticeable, needs a better explanation than implausible unfamiliarity with power functions in scientists with otherwise excellent quantitative skills. Allometric predictability leaves fundamental questions unanswered. Why should the cortex, or the forebrain and cerebellum together, have an overwhelming positive allometry across vertebrates? Within the cortex, why should the prefrontal cortex be advantaged whenever larger brains evolve across mammals? Without dismissing physical causes underlying scaling functions, let me offer examples of functional explanations for these cases which could hold across all species. For example, considering the first case, that when larger brains evolved independently in all major vertebrate lineages, extra neurons and/or process volume are routinely added preferentially to forebrain and cerebellum by their allometric scaling rules. Perhaps extra neurons are more likely to find useful employment if added to multimodal, plastic regions of the brain, where they can be dedicated to special functions as required, rather than having them added equally across all brain regions. In the familiar Darwinian argument, animals with this allometric rule simply survived and reproduced in greater numbers across all lineages and niches. At some point, such a generally adaptive solution might become a basic feature of the conserved vertebrate brain and body plan, whose active conservation over time requires a somewhat more elaborate account [Gerhart and Kirschner, 1997; Workman et al., 2013].

In the case of the prefrontal cortex, I suggest conceptualizing it as a structure whose inputs, outputs, and longterm memory capacity involves it in the integration of information for decisions over the whole duration of life, in multiple social conditions, and in changing physical spaces [see also Genovesio et al., 2014]. This combination of functions makes it particularly valuable in animals with extensive physical environments, multiple or complex social arrangements, and long lives. The strong positive correlations between duration of development, brain mass, and longevity suggest larger brains would be advantaged by having more relative volume given to the prefrontal cortex, which is simply a different way of phrasing "the prefrontal cortex should have positive allometry."

The correlations and interrelations of duration of neurodevelopment, brain mass and life history are numerous and diverse in mammals [Isler and van Schaik, 2012; Powell et al., 2017]. It takes longer to make a larger brain, and such animals require a longer period of brain organization after initial neurodevelopment [Workman et al., 2013; Halley and Deacon, 2017]. Parental care is of longer duration in animals with large brains, potentially offering more time to learn [Hawkes and Finlay, 2018]. As larger-brained animals statistically have larger bodies, they are likely to range larger physical spaces. Brain and body mass together are strongly positively correlated with lifespan [Hofman, 1993]. Positive allometry of the volume of the prefrontal cortex, which combines the properties of specialization for long-term memory, and the generation of decisions in the context of absolute physical space, social niche, and lifespan, should improve the survival and reproduction of larger-brained animals differentially. Its increasing relative size in animals with larger bodies and brains, and all the other features of life which covary with size, should be thought of as unremarkable and useful as the neurodevelopmental mechanisms which match an expected volume of the thalamus and somatosensory cortex to changing body surface areas. It is simply more abstract.

Some mammals might not maximally employ the neural space made available by prefrontal positive allometry, while other mammals might elaborate and substantially change its functionality. Important functional changes need not obviously change the borders or volumes of identified Brodmann areas in the frontal cortex. We have strong candidates for this type of evolutionary change in brains, which are the varying linkages between circulating hormones, neuromodulators and their receptors, and frontostriatal reward circuitry [Newman, 1999; Young and Wang, 2004; Goodson, 2008; Hofmann et al., 2014]. For example, a species might privilege social learning via such subcortical mechanisms resulting in an altered pattern of attention, and new learning patterns [Syal and Finlay, 2011]. An appropriately repurposed frontal cortex, instructed by new content, would be the result.

Before leaving this section, let me note an additional interesting feature of Figure 1, to which we will return. The allometry of individual variation (the approx. 60 individuals plotted per primate species) is identical in its equation to between-species allometry, a phenomenon described previously for other features of the human cortex [Toro et al., 2008, Charvet et al., 2013], and for individual variation of various features in a number of other mammals [Finlay et al., 2011].

\section{The Allometry of Developmental Duration and Life History}

The number of claims about the unusual duration of human development or unique interactions between brain growth and special human abilities is nearly as large 
Fig. 2. a The rate of progress for five species through the event scale of neurodevelopment as described in Workman et al. [2013]. The smallest values on the event scale are the first evidence of terminal neurogenesis in various spinal cord and medulla sites; the largest values are forebrain myelogenesis events. Cortical neurogenesis terminates at 0.50 , eye opening has the value 0.65 , and $80 \%$ of mature brain volume is found at 0.95 . The $y$-axis represents days of development on a linear scale. Circles and vertical dashed lines show the position of birth with respect to neural maturation in each species. $\mathbf{b}$ The differences in birth placement for humans, chimpanzees ( $P$. paniscus), and rhesus macaque. Redrawn from Finlay and Workman [2013].

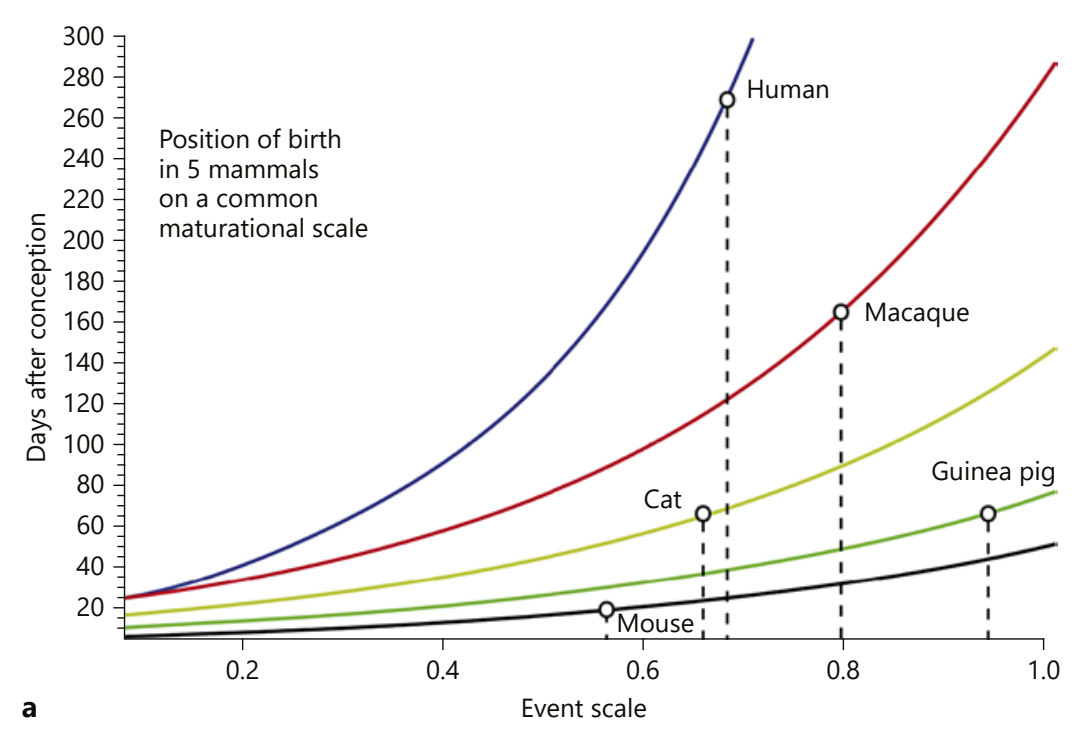

a

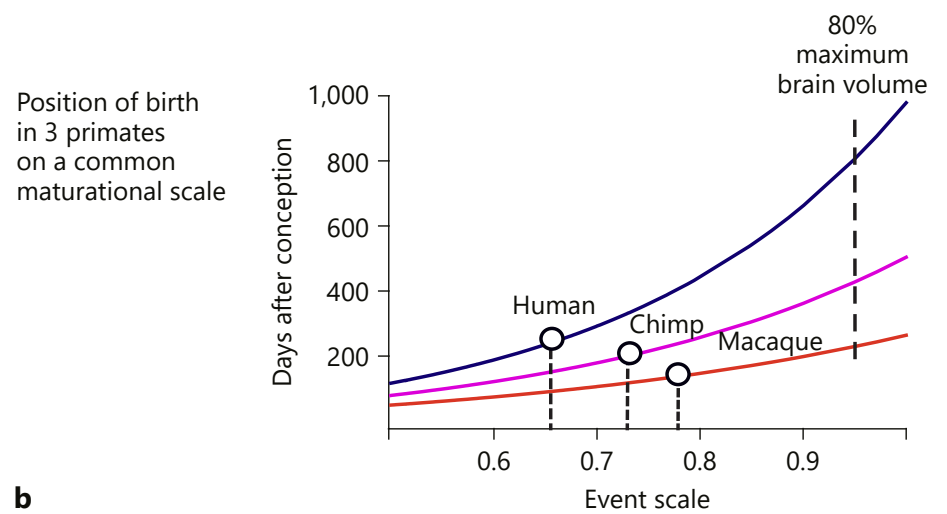

as the number of claims about the unique nature of the human cortex. The errors that are made are often parallel, including the presumption of linear scaling, or crediting features of development that are general to all mammals to humans alone. In addition, discussion of developmental allometry often stumbles over the discontinuity of birth, the timing of which varies widely with respect to brain maturation. Allometric approaches to development are somewhat less common than those describing neural mass. Our laboratory has developed a number of approaches to normalizing the progress of maturation across as many mammalian species as possible, which we have named "Translating Time." Space does not permit a detailed description of this work, and extended discussions may be found elsewhere [Workman et al., 2013; Finlay and Uchiyama, 2017; Hawkes and Finlay, 2018].
For an increasing number of species (18 fully modeled, and several others partially analyzed), neurodevelopmental events (over 251), and postnatal extent (up to 7-8 years of human postnatal equivalence), we have modeled the best order and interval relationship of the 251 events, including events like neurogenesis, myelination, attainment of specific fractions of adult brain volumes, and some behavioral milestones, called the "event scale" [Workman et al., 2013; www.translatingtime.org]. Using the computed event scale as the $x$-axis and post-conception day as the $y$-axis, each species' maturation can be represented by a regression equation (Fig. 2). The slope of each species' equation represents the rate of maturation as the number of days required to progress through the series of events on the event scale. The intercept approximates the initiation of neurogenesis. On such a plot, 
large-brained animals with long and slow maturation have steep slopes, requiring many days for each maturational step as they produce large volume changes at each one. Smaller-brained animals have flatter slopes, maturing quickly. The progress of maturation is highly conserved across species, well predicted on its appropriate log-transformed scale. Thus far, we have only found two interaction terms between neural structure, niche, or mammalian order which make a better fit of data to model. These two together account for about $6 \%$ of the total variance, bringing the overall fit of data to model to over $99 \%$. The first interaction term delays neurogenesis in the cortex of several mammalian orders, which amplifies neocortical volume over olfactory-limbic structures. The second delays neurogenesis in the retinas of nocturnal animals, which privileges the numbers of rods and rodrelated neurons of the retina.

Across species, various patterns in timing can be distinguished. Eutherian mammals produce neural tissue at a higher rate than marsupials, though both obey the same ordering of events [Darlington et al., 1999]. Altricial animals like mice and marsupials begin neurogenesis early, very soon after conception, while precocial animals like guinea pigs and sheep tend to delay neurodevelopment (primates are intermediate). No mammalian species studied to date is known to alter the rate of neurodevelopment (neither locally nor globally), and there is no evidence of any interruptions of the developmental sequence (birds can show such effects, however [Charvet and Striedter, 2010]). In most eutherian mammals, the duration of development is the single best predictor of brain size. However, some interesting deviations from this general rule are presently being investigated in marsupials [Darlington et al., 1999], bats [Martínez-Cerdeño et al., 2018], and naked mole rats [Buffenstein and Jarvis, 2002].

Our Translating Time model includes only neural events and a few early behaviors, initially intended as a tool to predict the timing of events in species for which data were not available. However, our model serves a second function. Life history events, like birth and weaning, puberty and other reproductive behavior transitions are more variable in their timing than the events we used to build our model initially, perhaps because of the conflicts and trade-offs over the duration and amount of resources contributed by parents to offspring. Although these events are not part of the model, the model can serve as an anchor for better and explicit comparison of life history events in different species. Birth is so variable with respect to the event scale, even within primates, that it serves as a very poor zero point for any maturational function (Fig. 2a, b). Conception is a better time axis origin, but the variability in neural maturation contributed by the precocial-altricial dimension makes the onset of neurodevelopment computed by the model best. In Figure $2 \mathrm{a}$, the start-to-end of neurodevelopment is made equivalent by the "event scale" $x$-axis for the five species represented, with absolute days of development on the $y$ axis, showing increasing maturational duration as steeper slopes to reach the developmental milestones specified on the $x$-axis. This kind of graph answers questions of the type: "If a mouse takes 5 days to generate its cortex, how many days does a rhesus macaque take?" Event-scale values for all 251 events can be found in Workman et al. [2013] or at www.translatingtime.org.

Applying estimation procedures derived from the Translating Time model to human and primate life history indicated in some cases that humans were well described as allometrically scaled non-human primates, but also produced some surprises [discussed at length in Finlay and Uchiyama, 2017; Hawkes and Finlay, 2018]. First, primate birth falls solidly in the middle of the overall mammalian range, with human birth moved a bit earlier on the neural event scale, but not dramatically so [Finlay and Workman, 2013] (Fig. 2b). A number of reports of developmental surges and rate changes in neural events like synaptogenesis or whole-structure volume growth in humans versus other primates are often the result of centering or starting maturational curves at birth, artificially offsetting maturational curves that would otherwise be essentially superimposable [Dobbing and Sands, 1979; Huttenlocher and Dabholkar, 1997; Leigh, 2004; Sakai et al., 2012a, b]. The observation that most volumetric growth of the human brain occurs after birth (in contrast to other primates) is seen in many developmental textbooks. The implied significance is that this postnatal growth is the reason for exceptional human learning and plasticity. This volume increase, however, has the prosaic explanation that the volume of myelinated tracts has a strong positive allometry with brain size. Most myelination is postnatal in all primates, but humans simply have more myelin, generating a greater post-birth volume change [Dobbing and Sands, 1979; Leigh, 2004]. Timeto-puberty and time-to-first-reproduction are quite variable both within and across primate species, but neither are obviously earlier or later in humans than what the Translating Time model would predict. Therefore, the overall duration of human infancy and childhood is disproportionately long, but predictable, in exactly the way that the disproportionate volume of prefrontal cortex is predictable. 
Fig. 3. Variability in weaning compared to neural maturational state. Predicted developmental schedules are shown for eight primate and seven non-primate species, with the observed timing of weaning indicated for each. The event scale on the $x$ axis has been extended beyond its original range of 0-1 to allow for extrapolation of developmental trajectories into the range in which weaning occurs. Examples of events included in the model are displayed at their respective positions on the event scale on the $x$-axis. The $y$-axis indicates the estimated date of the occurrence of each event in each species. Species are as follows: baboon (P. cynocephalus); cat (F. catus); chimpanzee (P. troglodytes); ferret (M. putorius); gerbil (M. unguiculatus); guinea pig (C. porcellus); hamster (M. auratus); human (H. sapiens); lemur (L. catta); macaque (M. mulatta); marmoset (C. jacchus); Orangutan (P. pygmaeus pygmaeus); owl monkey (A. azarae); rabbit (O. cuniculus); rat (R. norvegicus); sheep (O. aries). Redrawn from Finlay and Uchiyama [2017].

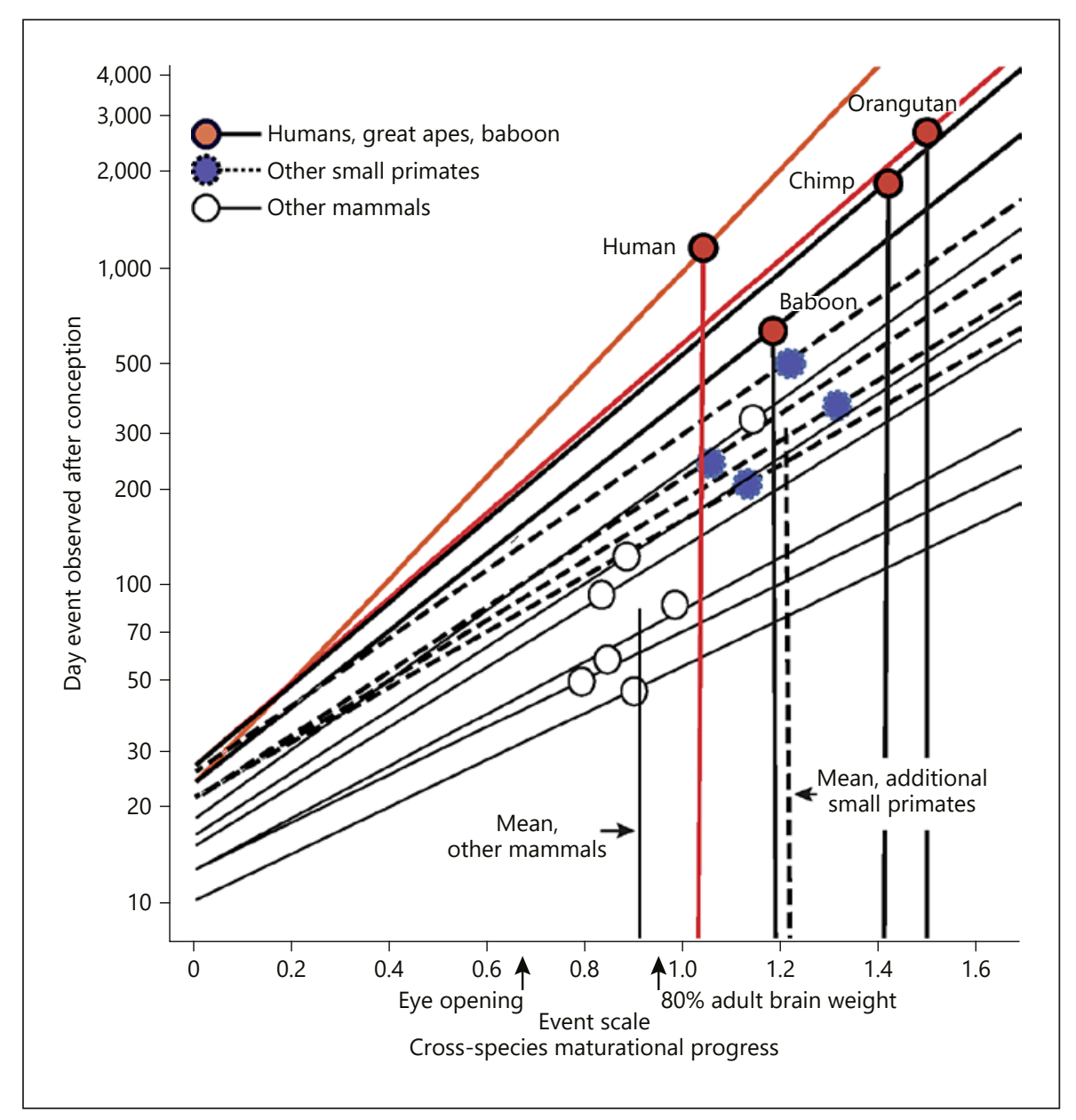

The biggest surprise in our analysis of human life history is weaning. As most anthropologists know well [Galdikas and Wood, 1990; Hawkes et al., 1998], but psychologists and neurobiologists tend to get wrong, humans wean their infants markedly earlier than do the other great apes (Fig. 3). Using Translating Time for explicit scaling, if humans weaned their infants on the chimpanzee schedule, they would do so five or more years after birth. Thus, humans clearly reduced their minimal interbirth interval, which explains the impressive increase in fecundity of humans, compared to other great apes [Jones et al., 1999].

Early weaning virtually requires the aid of others. If a human mother turns her attention to a new infant, it is advantageous to have someone else take over part of the protection and provisioning of the under-5-year-old toddler. The help of siblings and extended family, paternal care in many cases, and most notably the assistance of grandmothers, are all available for the care of infants not yet able to forage on their own [Hawkes et al., 1998, Hrdy, 2011]. Such extensive social support and provisioning by non-mothers is rarely seen in other great apes [Hrdy, 2011]. The existence of grandmothers themselves (i.e., non-senescent, knowledgeable individuals uninvolved with the care of their own infants) is made possible by a complementary alteration of life history at the end of human life, menopause, which is also not seen in other apes.

Overall, appropriate normalization of life history with respect to brain development erases claims of bumps and accelerations in brain growth in support of human-specific traits like early language acquisition. It also lends support to the increasingly appreciated view that, given our large brains, the key evolutionary change in early human ontogeny is driven by its social context [Sterelny, 2017]. By comparing developmental time allometrically, we come to see how the intense sociality of humans, as it first appears in support of infants by multiple individuals, supports cultural learning [Boyd et al., 2011]. 


\section{Mouse Exceptionalisms, Unintended}

Model species tend to have a convenient size, rapid reproduction and other laboratory conveniences, and are expected not to be specialists with respect to the human traits they are expected to model. To a degree, whether an animal is a specialist or generalist does not matter. The overarching reason that $M$. musculus allows us to understand brain organization is the same one that allows Aplysia, Drosoph$i l a$, and $D$. rerio their continued usefulness: the conservation of fundamental developmental processes at the cellular level, the conserved body plan spanning all three phyla, and fundamental features of physiology specific to neurons. Conserved developmental programs and cellular mechanisms are those that permit robust and stable variations, not ones that dictate adult characters; so adaptive specializations in particular animals should not thwart research on species commonalities, so long as we have a wellinformed view of the range of variations possible.

More than a century of research has turned up unpredicted, unusual features in mouse sensory organization compared to other mammals. These features raise questions about how well we understand actual variation in brain and sensory systems, what we predict, and why we predict it. Not only that, in their years of service as model systems, some mouse strains appear to have accrued peculiarities via "evolutionary" processes operating in a laboratory setting. Here, I describe some of these mouse exceptionalisms and consider which might be cause for concern.

\section{Unusual Specializations}

Because of its workhorse role as a model species, the mouse has been subjected to close investigations of the functional organization of all of its sensory systems that might not have been undertaken otherwise. Investigation of the visual and auditory systems have disclosed two unusual features, and in both cases, the feature is present both in field M. musculus and the lab mouse.

First, though mice are nocturnal, and rods predominate in their retinas, mice do possess a cone photoreceptor, and two cone opsins. The mouse photoreceptor and its opsins are unusual in two ways compared to general expectations arising from research in primates. First, single photoreceptors in mice may express both opsins (primate photoreceptors express only one). Second, the opsins in mice have an unusual retinal distribution: short wavelength, UV-sensitive opsins are found in the ventral retina, whereas middle-wavelength opsins are located in the dorsal retina. More accurately, the two opsins are ex- pressed in opposing dorsal-ventral gradients, with cones in the middle retina expressing both [Applebury, 2001; Neitz and Neitz, 2001]. Similar distribution gradients, the functional significance of which remains unclear, have subsequently been described also in some other rodent species. As the discoverers of this unusual organization were quick to point out, this organization can serve as a feature rather than a bug in that it offers the opportunity to explore molecular mechanisms of opsin expression. In any case, it is useful to know that mouse retinas are distinct from primate retinas in these two features associated with diurnality.

The auditory system is also unique. In an unusual kind of quantitative study, Glendenning and Masterton [1998] characterized the subcortical auditory system in fiftythree diverse mammals, measuring the volume of ten auditory nuclei, ranging from the cochlear nuclei to the medial geniculate body of the thalamus. The sample set including bats, primates (including humans), rodents, marsupials, and more. For each distribution of nucleus volumes, a $z$-score can be calculated, and summed over all ten nuclei to make a score of greatest deviation from the fifty-three-animal mean (as distinct from the relatively smallest or largest). On that scale, the mouse is the most deviant, followed by the little brown bat and the kangaroo rat. The nucleus making the greatest contribution to the deviance score of mice is their unusually large superior olive; the functional significance of this variation is entirely unknown.

These two observations might well take any researcher aback, not because they necessarily do damage to the mouse's status as a model species, but because they underline our lack of knowledge of the normal deviations in volume, neuron number, and composition in sensory, motor, and sensory systems across species, and most importantly, the functional significance of those deviations. The idea that neural tissue should be preferentially dedicated to the circuitry most useful to the animal is so established that it has a name, the theory of "proper mass" [Jerison, 1973]. Jerison and subsequent researchers focused on one dimension of variation in niche seen across all vertebrates: olfactory versus visual specialists can be distinguished by the relative volumes of their central nervous system olfactory and visual structures [Barton, 2004; Yopak et al., 2010]. Development tells a confirming mechanistic story: brain mass is allocated during early development preferentially to olfactory-limbic versus restof-brain circuitry depending on niche by means of change in duration of neurogenesis [Finlay and Darlington, 1995; Reep et al., 2007; Workman et al., 2013]. 
Other than the olfactory-limbic versus visual-isocortex axis, however, it has been surprisingly difficult to detect further candidates for changed proper mass allocation in mammalian brains (that is, altered neural mass proportions via genetic specification of new structure boundaries or changes in rate or duration of neurogenesis). With appropriate allometric scaling, the shared predictable variation in brain volume across mammals is very high, in the range of 95-99\%, giving little evidence of genetically directed volume redistribution [Aboitiz et al., 2003; Kaskan et al., 2005]. By contrast, small but robust volume changes in cortical areas are a common outcome of differential experience (as distinct from genetic specification), both in the lab and in the field [Krubitzer and Prescott, 2018]. In fact, "proper mass" may typically be realized by functional repurposing of neurons within cortical areas [Anderson and Finlay, 2014]. Researchers may fail to appreciate the ubiquity of repurposing or reuse because of the assumption of fixed and single functions in our naming conventions for brain parts. For example, the names "primary visual cortex," "V1," or "striate cortex" (not unreasonably) were given to the conspicuous striated area of occipital cortex that receives its primary thalamic input from the lateral geniculate body by neuroanatomists, but meta-analyses of thousands of imaging studies show that V1 is routinely activated, as are all primary sensory areas, by other sensory, motor, and cognitive tasks [Anderson, 2010, 2014]. The same large databases show that the canonical "language" area, "Broca's area," is more likely to be activated by nonlanguage than language tasks [Poldrack, 2011].

The intent of this section was, first, to highlight two unexpected features of the mouse visual system and auditory system, and second, to inquire why we find the mouse data surprising. In the auditory system case, the mouse showed high variance and an unusually large olivary nucleus. The latter was a surprise because the mouse, not known to be an auditory specialist, was not expected to have any unusually large auditory system components. We went on to argue that our understanding of functional allocation may as yet be very uninformed, biased by the way we normally label and discuss brain parts. These considerations should make us careful about what we presume to be conserved between both M. musculus and $H$. sapiens.

\section{Unusual Allometry in Some Mouse Strains}

In order for allometric scaling of brain components across species to emerge as an evolutionary phenomenon, evidence of the same scaling should be observed in indi- vidual variation within species, as heritable individual variation is what permits natural selection. Although the data for the allometric scaling of the prefrontal cortex in rhesus, chimpanzee, and human in Donahue et al. [2018] was not gathered to demonstrate this assertion, its pattern is easily observed (Fig. 1): for every cortical area measured, the scaling of cortical area volumes in individual animals falls close to the cross-species regression line. We have observed the same relationship between individual and cross-species variation in the allometry of gross brain divisions, as measured in a variety of domesticated species [Finlay et al., 2011] and also in humans [Charvet et al., 2013]. The same assumption proves useful in modeling details of cortical organization [Cahalane et al., 2014]. This hypothesis works well for allometric scaling patterns that are conserved across species, but can the same relationship of individual to cross-species variation be observed for dimensions that differentiate species?

The allocation of volume across gross brain divisions in all vertebrates exhibits a two-factor structure, allocating tissue differentially to olfactory-limbic structures versus either the rest-of brain or isocortex [Reep et al., 2007; Yopak al., 2010]. Diurnal primates load high on factor 1 and olfactory-specialist anteaters load high on the olfactory factor 2. Deep-sea sharks have bigger olfactory bulbs than those that live in brightly-lit shallows. If similar kinds of variation appear at the species level across the vertebrate radiations, there must be within-species, individual variation to support it. So, does the pervasive pattern of cross-species variation also appear in the withinspecies variation, as relative loadings on factor 1 (whole brain minus olfactory-limbic, or cortex) versus factor 2 (olfactory-limbic; Fig. 4)?

To investigate this question, we made use of Kruska's careful delineation of major brain regions in 36 individual pigs and minks [Plogmann and Kruska, 1990; Kruska, 1996], originally gathered to look at the effects of domestication. We also looked at 11 commonly used mouse strains, analyzing each inbred strain as if it were an individual [Finlay et al., 2011]. In a later study (not pictured in Fig. 4), we investigated the same kind of individual variation in humans. Figure 4 a shows the pattern of loading on gross brain structures for factors 1 and 2 across mammals, and Figure $4 \mathrm{~b}$ shows the overall pattern of loading on the same structures for the 48 pigs and minks, and the 11 mouse strains. The pattern of cross-species variation was nearly identical to the within-species loading on factors 1 and 2. The everyday significance of observation is that there exist naturally high-olfactory or low-isocortex individuals in pigs and minks, and in hu- 


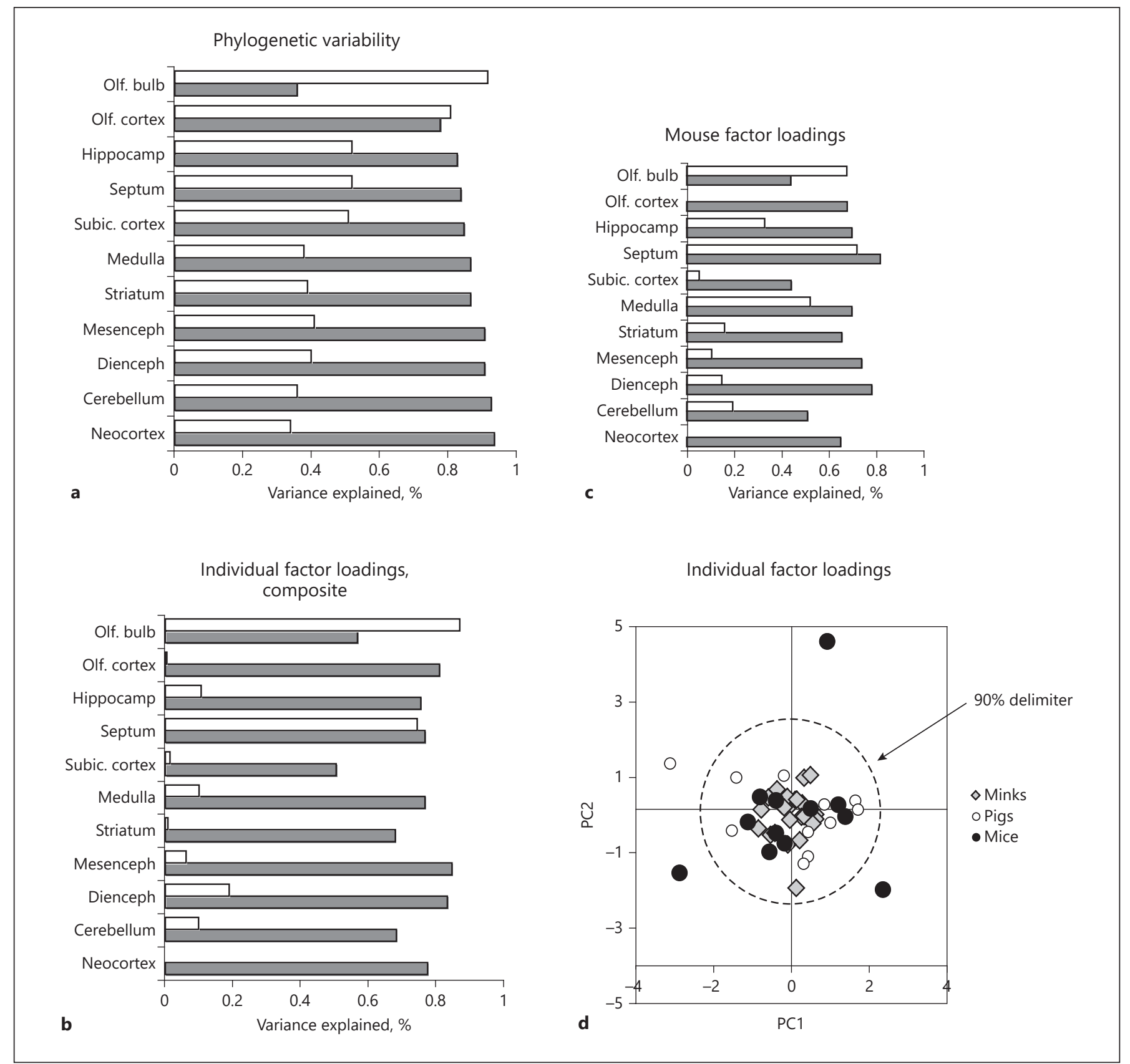

Fig. 4. a-c Percentage of variance in each structure captured by the first principal component (PC1) is represented by the black bars, and the second principal component (PC2) is represented by the white bars. In all three graphs, PC1 accounts for a much greater amount of variance in the volumes of neural structures than PC1. The length of each bar represents the amount each neural structure contributes to the "percent variance explained" for PC1 and PC2, respectively, noting again that PC2 is a much smaller contributor to overall variance than PC1. a "Neocortex" is the highest contributing structure to PC1 and "olfactory bulb" to PC2 . The illustrated "phylogenetic variability" is based on a sample of 131 species of bats, primates, and insectivores. PC1 accounts for $96.47 \%$ and PC2 for $2.61 \%$ of the overall variation in structure volumes. $\mathbf{b}$ This measure of "individual vari-

ability, composite" is based on 47 individuals whose scores were entered as deviations from overall means so as to exclude species, sex, and domestication differences; the data were derived from 6 male wild mink, 6 female wild mink, 6 male domestic mink, 6 female domestic mink, 6 wild pigs of unknown sex, 6 domestic pigs of unknown sex, and 11 mouse strains. PC1 accounts for $72.48 \%$ of the variance, and PC2 for 7.9\%. c For the mouse, data are plotted so that their overall pattern might be examined, but no statistical claims about factor loadings on individual structures can be made. $\mathbf{d}$ Values of PC1 and PC2 are expressed as $z$-scores for each individual animal for the population of 47 individuals. Three of the mice and 1 pig fall outside the $90 \%$ delimiter (dashed line); these mouse strains are WSB/ei, MOLC/ei, and MOLf/ei. Redrawn from Finlay et al. [2011]. 
mans as well. Presumably these individuals provide a hypothesized source of variation for ongoing selection.

If the mouse strains are separated from the pigs and minks, however, their loading pattern is ordered differently (Fig. 4c). Figure 4d demonstrates a source of the disorder - of the 47 individuals analyzed, 1 pig and 3 of the 11 mouse strains fall outside of the $90 \%$ bounds for loading on these two factors for this sample, with each of the 3 strains deviating in a different manner. That some laboratory mice might fail to exhibit a pattern of variation that has been established in vertebrates for some 450 million years is disconcerting. Since duration of neurogenesis is a function of combined anterior/posterior and medial/ lateral position in the embryonic neural plate, the lack of this correspondence suggests a possible defect in one of the most basic vertebrate organizational features, the conserved body and brain plan [Gerhart and Kushner, 1997]. Perhaps these mouse strains could be a source of insight to the outcomes of such a genetic defect, but they are far from normal and should probably be disqualified for use as models for any type of functional organization.

\section{Conclusion}

This review has offered several examples of premature or mistaken claims about human uniqueness, as well as causes for concern about the assumed typicality of the mouse model. More generally, we have looked at assump- tions about predictability and typicality about the brains of different species, and how they might be verified. In most cases, these problems can be corrected by employing available information to investigate the empirical assertions and to understand where allometric analyses suggest we should look. Neuroscience itself, with evolutionary neuroscience as a component, has a unique evolutionary history. The extreme positive allometry of the field of neuroscience over the past 50 years has exploded the information available to neuroscience, making it difficult to absorb new information, let alone reconsider established paradigms. Rare symposia of the kind represented by this volume allow an invaluable opportunity to evaluate where our initial choices have led us, and where we might choose to go next.

\section{Acknowledgements}

I would like to thank all my colleagues and coworkers whose laboratory work and insights represented in our jointly published work cited here form the core of this review, namely Richard Darlington, Christine Charvet, Flora Hinz, Alan Workman, Diarmuid Cahalane, Barbara Clancy, Luiz Carlos de Lima Silveira, Isle Bastille, Ryutaro Uchiyama, Peter Kaskan, Sarah Dunlop, Elizabeth Yamada, and Edna Christina Franco.

\section{Disclosure Statement}

The author has no conflicts of interest to declare.

\section{References}

Aboitiz F, Morales D, Montiel J. The evolutionary origin of the mammalian isocortex: towards an integrated developmental and functional approach. Behav Brain Sci. 2003 Oct;26(5): 535-52.

Anderson ML. Neural reuse: a fundamental organizational principle of the brain. Behav Brain Sci. 2010 Aug;33(4):245-66.

Anderson ML. After phrenology: neural reuse and the interactive brain. Cambridge: MIT Press; 2014. https://doi.org/10.7551/mitpress/10111.001.0001.

Anderson ML, Finlay BL. Allocating structure to function: the strong links between neuroplasticity and natural selection. Front Hum Neurosci. 2014 Jan;7:918.

Applebury ML. Response: the uncommon retina of the common house mouse. Trends Neurosci. 2001;24(5):250.

Barton RA. From The Cover: binocularity and brain evolution in primates. Proc Natl Acad Sci USA. 2004 Jul;101(27):10113-5.
Barton RA, Montgomery SH. Proportional versus relative size as metrics in human brain evolution. Proc Natl Acad Sci USA. 2019 Jan; 116(1):3-4.

Barton RA, Venditti C. Human frontal lobes are not relatively large. Proc Natl Acad Sci USA. 2013 May;110(22):9001-6.

Bowmaker JK. Evolution of colour vision in vertebrates. Eye (Lond). 1998;12(3 Pt 3b):541-7.

Boyd R, Richerson PJ, Henrich J. The cultural niche: why social learning is essential for human adaptation. Proc Natl Acad Sci USA. 2011 Jun;108(Suppl 2):10918-25.

Buffenstein R, Jarvis JU. The naked mole rat - a new record for the oldest living rodent. Sci Aging Knowledge Environ. 2002;2002(21): pe7.

Cahalane D J, Charvet CJ Finlay BL. Modeling local and cross-species neuron number variations in the cerebral cortex as arising from a common mechanism. Proc Natl Acad Sci USA. 2014;111(49):17642-7.
Capranica RR, Rose G (Huber F, Markl H, editors). Frequency and temporal processing in the auditory system of anurans. Neuroethology and Behavioral Physiology. Berlin, Heidelberg: Springer; 1983. pp. 136-52.

Charvet CJ, Darlington RB, Finlay BL. Variation in human brains may facilitate evolutionary change toward a limited range of phenotypes. Brain Behav Evol. 2013;81(2):74-85.

Charvet CJ, Finlay BL. Comparing adult hippocampal neurogenesis across species: translating time to predict the tempo in humans. Front Neurosci. 2018 Oct;12:706.

Charvet CJ, Striedter GF. Bigger brains cycle faster before neurogenesis begins: a comparison of brain development between chickens and bobwhite quail. Proc Biol Sci. 2010 Nov; 277(1699):3469-75.

Darlington RB, Dunlop SA, Finlay BL. Neural development in metatherian and eutherian mammals: variation and constraint. J Comp Neurol. 1999 Aug;411(3):359-68. 
Dobbing J, Sands J. Comparative aspects of the brain growth spurt. Early Hum Dev. 1979 Mar;3(1):79-83.

Donahue CJ, Glasser MF, Preuss TM, Rilling JK, Van Essen DC. Quantitative assessment of prefrontal cortex in humans relative to nonhuman primates. Proc Natl Acad Sci USA. 2018 May;115(22):E5183-92.

Doupe AJ, Kuhl PK. Birdsong and human speech: common themes and mechanisms. Annu Rev Neurosci. 1999;22(1):567-631.

Finlay BL. The developing and evolving retina: using time to organize form. Brain Res. 2008 Feb;1192:5-16.

Finlay BL, Charvet CJ, Bastille I, Cheung DT, Muniz JA, de Lima Silveira LC. Scaling the primate lateral geniculate nucleus: niche and neurodevelopment in the regulation of magnocellular and parvocellular cell number and nucleus volume. J Comp Neurol. 2014 Jun; 522(8):1839-57.

Finlay BL, Cheung D, Darlington RB. Developmental constraints on or developmental structure in brain evolution? In: Munakata Y Johnson M, editors. Processes of change in brain and cognitive development. New York: Oxford University Press; 2005. p. 128-62.

Finlay BL, Darlington RB. Linked regularities in the development and evolution of mammalian brains. Science. 1995 Jun;268(5217):157884.

Finlay BL, Darlington RB, Nicastro N. Developmental structure in brain evolution. Behav Brain Sci. 2001 Apr;24(2):263-78.

Finlay BL, Hinz F, Darlington RB. Mapping behavioural evolution onto brain evolution: the strategic roles of conserved organization in individuals and species. Philos Trans R Soc Lond B Biol Sci. 2011 Jul;366(1574):2111-23.

Finlay BL, Uchiyama R. The timing of brain maturation, early experience, and the human social niche. In: Kaas J, editor. Evolution of Nervous Systems 2e. Volume 3. Oxford: Elsevier; 2017. pp. 123-48. ISBN: 9780128040423.

Finlay BL, Workman AD. Human exceptionalism. Trends Cogn Sci. 2013 May;17(5):199201.

Galdikas BM, Wood JW. Birth spacing patterns in humans and apes. Am J Phys Anthropol. 1990 Oct;83(2):185-91.

Genovesio A, Wise SP, Passingham RE. Prefrontal-parietal function: from foraging to foresight. Trends Cogn Sci. 2014 Feb;18(2):72-81.

Gerhart J, Kirschner M. Cells, embryos and evolution. Malden: Blackwell Science; 1997.

Gibson WT, Gonzalez CR, Fernandez C, Ramasamy L, Tabachnik T, Du RR, et al. Behavioral responses to a repetitive visual threat stimulus express a persistent state of defensive arousal in Drosophila. Curr Biol. 2015 Jun; 25(11):1401-15.

Glendenning KK, Masterton RB. Comparative morphometry of mammalian central auditory systems: variation in nuclei and form of the ascending system. Brain Behav Evol. 1998; 51(2):59-89.
Goodson JL. Nonapeptides and the evolutionary patterning of sociality. Prog Brain Res. 2008; 170:3-15.

Greenough WT, Bailey WT. The anatomy of a memory: convergence of results across a diversity of tests. Trends Neurosci. 1988;11(4): 142-7.

Halley A, Deacon T. The developmental basis of evolutionary trends in primate encephalization. In: Kaas J, editor. Evolution of nervous systems. 2nd ed; vol. 3. Oxford: Elsevier; 2017. pp. 101-22.

Hauser MD, Chomsky N, Fitch WT. The faculty of language: what is it, who has it, and how did it evolve? Science. 2002 Nov;298(5598):156979.

Hawkes K, Chisholm JS, Fairbanks LA, Johow J, Kalcher-Sommersguter E, Liebal K, et al. Primate infancies: Causes consequences of varying care The Cultural Nature of Attachment: Contextualizing Relationships and Development Keller H Bard KA Cambridge. MA: MIT Press; 2017.

Hawkes K, Finlay BL. Mammalian brain development and our grandmothering life history. Physiol Behav. 2018 Sep;193(Pt A):55-68.

Hawkes K, O'Connell JF, Jones NG, Alvarez H, Charnov EL. Grandmothering, menopause, and the evolution of human life histories. Proc Natl Acad Sci USA. 1998 Feb;95(3): 1336-9.

Healy SD, Rowe C. A critique of comparative studies of brain size. Proc Biol Sci. 2007 Feb; 274(1609):453-64.

Hisey E, Kearney MG, Mooney R. A common neural circuit mechanism for internally guided and externally reinforced forms of motor learning. Nat Neurosci. 2018 Apr;21(4):58997.

Hofman MA. On the evolution and geometry of the brain in mammals. Prog Neurobiol. 1989; 32(2): 137-58.

Hofman MA. Encephalization and the evolution of longevity in mammals. J Evol Biol. 1993; 6(2):209-27.

Hofmann HA, Beery AK, Blumstein DT, Couzin ID, Earley RL, Hayes LD, et al.; NESCent Working Group on Integrative Models of Vertebrate Sociality: Evolution, Mechanisms, and Emergent Properties. An evolutionary framework for studying mechanisms of social behavior. Trends Ecol Evol. 2014 Oct;29(10): 581-9.

Hopkins CD. Evolution of electric communication channels of mormyrids. Behav Ecol Sociobiol. 1980;7(1):1-13.

Hoyle G. The scope of neuroethology. Behav Brain Sci. 1984;7(03):367-81.

Hrdy SB. Mothers and others: the evolutionary origins of mutual understanding. Cambridge (MA): Harvard University Press; 2011.

Huttenlocher PR, Dabholkar AS. Regional differences in synaptogenesis in human cerebral cortex. J Comp Neurol. 1997 Oct;387(2):16778.
Isler K, van Schaik CP. Allomaternal care, life history and brain size evolution in mammals. J Hum Evol. 2012 Jul;63(1):52-63.

Jarvis ED, Güntürkün $\mathrm{O}$, Bruce L, Csillag A, Karten H, Kuenzel W, et al.; Avian Brain Nomenclature Consortium. Avian brains and a new understanding of vertebrate brain evolution. Nat Rev Neurosci. 2005 Feb;6(2):151-9.

Jerison HJ. Evolution of the Brain and Intelligence. New York: Academic Press; 1973.

Jones NB, Hawkes K, O'Connell JF. Some current ideas about the evolution of the human life history. In: Lee PC, editor. Comparative primate socioecology. Cambridge: Cambridge University Press; 1999. p. 140-66.

Kaskan PM, Franco EC, Yamada ES, Silveira LC, Darlington RB, Finlay BL. Peripheral variability and central constancy in mammalian visual system evolution. Proc Biol Sci. 2005 Jan; 272(1558):91-100.

Krubitzer LA, Prescott TJ. The combinatorial creature: cortical phenotypes within and across lifetimes. Trends Neurosci. 2018; 41(10):744-62.

Kruska D. The effect of domestication on brain size and composition in the mink (Mustel vison). J Zool (Lond). 1996;239(4):645-61.

Lande R. Quantitative genetic analysis of multivariate evolution, applied to brain:body size allometry. Evolution. 1979 Mar;33(1Part2): $402-16$.

Lefebvre L. Brains, innovations, tools and cultural transmission in birds, non-human primates, and fossil hominins. Front Hum Neurosci. 2013 Jun; 7:245.

Lefebvre L, Reader SM, Sol D. Brains, innovations and evolution in birds and primates. Brain Behav Evol. 2004;63(4):233-46.

Leigh SR. Brain growth, life history, and cognition in primate and human evolution. Am J Primatol. 2004 Mar;62(3):139-64.

MacLean EL, Hare B, Nunn CL, Addessi E, Amici $\mathrm{F}$, Anderson RC, et al. The evolution of selfcontrol. Proc Natl Acad Sci USA. 2014 May; 111(20):E2140-8.

Martínez-Cerdeño V, Camacho J, Ariza J, Rogers H, Horton-Sparks K, Kreutz A, et al. The bat as a new model of cortical development. Cereb Cortex. 2018 Nov;28(11):3880-93.

Nauta WJ, Karten HJ. A general profile of the vertebrate brain, with sidelights on the ancestry of the cerebral cortex Neuroanatomy Boston. MA: Birkhauser; 1970. pp. 520-39.

Nave G, Jung WH, Karlsson Linnér R, Kable JW, Koellinger PD. Are bigger brains smarter? Evidence from a large-scale preregistered study. Psychol Sci. 2019;30(1):43-54.

Neitz M, Neitz J. The uncommon retina of the common house mouse. Trends Neurosci. 2001 May;24(5):248-50.

Newman SW. The medial extended amygdala in male reproductive behavior. A node in the mammalian social behavior network. Ann NY Acad Sci. 1999 Jun;877:242-57.

Northcutt RG. Evolution of the telencephalon in nonmammals. Annu Rev Neurosci. 1981; $4(1): 301-50$ 
Nottebohm F, Stokes TM, Leonard CM. Central control of song in the canary, Serinus canarius. J Comp Neurol. 1976 Feb;165(4):457-86.

Passingham RE, Smaers JB. Is the prefrontal cortex especially enlarged in the human brain allometric relations and remapping factors. Brain Behav Evol. 2014;84(2):156-66.

Plogmann D, Kruska D. Volumetric comparison of auditory structures in the brains of European wild boars (Sus scrofa) and domestic pigs (Sus scrofa f. dom.). Brain Behav Evol. 1990;35(3):146-55.

Poeppel D. The neuroanatomic and neurophysiological infrastructure for speech and language. Curr Opin Neurobiol. 2014 Oct;28: 142-9.

Poldrack RA. Inferring mental states from neuroimaging data: from reverse inference to largescale decoding. Neuron. 2011 Dec;72(5):6927.

Powell LE, Isler K, Barton RA. Re-evaluating the link between brain size and behavioural ecology in primates. Proc Biol Sci. 2017 Oct; 284(1865):24-53.

Quiring R, Walldorf U, Kloter U, Gehring WJ. Homology of the eyeless gene of Drosophila to the Small eye gene in mice and aniridia in humans. Science. 1994 Aug;265(5173):785-9.

Rasika S, Nottebohm F, Alvarez-Buylla A. Testosterone increases the recruitment and/or survival of new high vocal center neurons in adult female canaries. Proc Natl Acad Sci USA. 1994 Aug;91(17):7854-8.
Reep RL, Finlay BL, Darlington RB. The limbic system in mammalian brain evolution. Brain Behav Evol. 2007;70(1):57-70.

Remtulla S, Hallett PE. A schematic eye for the mouse, and comparisons with the rat. Vision Res. 1985;25(1):21-31.

Sakai T, Hirata S, Fuwa K, Sugama K, Kusunoki $\mathrm{K}$, Makishima H, et al. Fetal brain development in chimpanzees versus humans. Curr Biol. 2012a Sep;22(18):R791-2.

Sakai T, Matsui M, Mikami A, Malkova L, Hamada Y, Tomonaga M, et al. Developmental patterns of chimpanzee cerebral tissues provide important clues for understanding the remarkable enlargement of the human brain. Proc Biol Sci. 2012b;280:20122398.

Sheehan MJ, Tibbetts EA. Specialized face learning is associated with individual recognition in paper wasps. Science. 2011;334(6060): 1272-5.

Sol D, Duncan RP, Blackburn TM, Cassey P, Lefebvre L. Big brains, enhanced cognition, and response of birds to novel environments. Proc Natl Acad Sci USA. 2005 Apr;102(15):5460-

Sterelny K. Humans as model organisms. Proc Biol Sci. 2017;284(1869):2115.

Stevens JR. Evolutionary pressures on primate intertemporal choice. Proc Biol Sci. 2014; 281(1786):0499.
Suga N. Principles of auditory information-processing derived from neuroethology. J Exp Biol. 1989;146:277-86.

Syal S, Finlay BL. Thinking outside the cortex: social motivation in the evolution and development of language. Dev Sci. 2011 Mar; 14(2): 417-30.

Toro R, Perron M, Pike B, Richer L, Veillette S, Pausova Z, et al. Brain size and folding of the human cerebral cortex. Cereb Cortex. 2008 Oct;18(10):2352-7.

Vallender EJ. Exploring the origins of the human brain through molecular evolution. Brain Behav Evol. 2008;72(2):168-77.

West GB, Brown JH, Enquist BJ. A general model for the origin of allometric scaling laws in biology. Science. 1997 Apr;276(5309):122-6.

Wilsch-Bräuninger $\mathrm{M}$, Florio $\mathrm{M}, \mathrm{Huttner} \mathrm{WB}$. Neocortex expansion in development and evolution - from cell biology to single genes. Curr Op Neurobiol. 2016:39:122-32.

Workman AD, Charvet CJ, Clancy B, Darlington RB, Finlay BL. Modeling transformations of neurodevelopmental sequences across mammalian species. J Neurosci. 2013 Apr;33(17): 7368-83.

Yopak KE, Lisney TJ, Darlington RB, Collin SP, Montgomery JC, Finlay BL. A conserved pattern of brain scaling from sharks to primates. Proc Natl Acad Sci USA. 2010 Jul;107(29): 12946-51.

Young LJ, Wang Z. The neurobiology of pair bonding. Nat Neurosci. 2004 Oct; 7(10):104854 . 